Mon. Not. R. Astron. Soc. 000, 16 (0000) Printed 2 October $2018 \quad$ (MN LATEX style file v2.2)

\title{
A New Titanium Oxide Index in Visual Band
}

\author{
Bahar Bidaran ${ }^{1 \star}$, Mohammad Taghi Mirtorabi ${ }^{1}$ and Fatemeh Azizi ${ }^{2}$ \\ ${ }^{1}$ Department of Physics, Alzahra University, P.O. Box 1993893973, Tehran, Iran \\ ${ }^{2}$ Department of Physics, Payame Noor University (PNU), P.O. Box 19395-3697, Tehran, Iran
}

2 October 2018

\begin{abstract}
We introduce a new color index consisting of two spectral band width to measure $\mathrm{TiO}$ absorption band strength centered at $567 \mathrm{~nm}$. Based on the most up-to-date line list for $\mathrm{TiO}$ we regenerate a grid of synthesized spectra and investigate the temperature sensitivity of the index. The new index behave similar to older Wing TiO-index where it decrease monotonically from coolest atmosphere with $T_{\text {eff }}=2800$ up to $T_{\text {eff }}=4000$ where the $\mathrm{TiO}$ molecules disassociate. To further examine the feasibility of the new index we reproduce the calibration using a list of observed high resolution spectra and found similar results. This index extend $\mathrm{TiO}$ absorption band capability to measure effective temperature of late $\mathrm{K}$ to $\mathrm{M}$ stars to visual spectrum where it is more accessible to small telescopes for long term dedicated observation.
\end{abstract}

Key words: line formation - molecular data - late-type stars - instrumentation:spectrograph.

\section{INTRODUCTION}

' Molecular absorption is a signature of cool atmosphere in late type stars and magnetized active regions in $\mathrm{F}$ - K main sequences. They are consisting of wide band absorptions, ' observable with low resolution spectrographs or simple photometers attached to small telescopes. In a cool star atmo' sphere, atomic lines are weaker because of atomic depletion ' by molecules or grains formation or lower atomic states population. Due to physical conditions such as low temperature ' and low density which can cause infrequent collisions, the local density of molecules such as $\mathrm{ZrO}, \mathrm{CO}, \mathrm{TiO}$ and $\mathrm{VO}$ grow in cool atmospheres, because they survive from thermal dissociation (Robinson 2007). Molecular absorption may influence the spectrum as an individual lines or suppress the continuum in a wide interval of wavelengths which can change the whole structure of the spectrum. These pervasive spectral features have been used as a major characteristic of cool atmospheres to classify late type M stars (Ramsey 1981).

Among molecules mentioned above, TiO comes across as special one due to its wide and strong absorption in near infra-red. Its absorption bands start appearing and deepening in late $K$ and prevail until coolest $M$ type stars. In order to study cool stars using TiO, Wing has introduced a photometric system consists of three filters which are tuned to measure TiO $\gamma(1,1)$ R-branch band head at $719 \mathrm{~nm}$ on near infra-red (Wing 1992). He proposed this region because it was accessible to the solid state photometers at the time. This photometric system was used

* E-mail: b.bidaran@student.alzahra.ac.ir (BB) to study the correlation between choromospheric activity and $\mathrm{TiO}$ absorption strength in variable star $\lambda$ Andromeda (Mirtorabi, Wasatonic \& Guinan 2003). They have found an estimated activity cycle of about 4 to 14 years and an anti-correlation between average $\mathrm{TiO}$ absorption representing coverage of spots with average visual brightness; similar to what is observed in the Sun. The most updated continuum band-passes of this system has been published recently by Azizi \& Mirtorabi (2015). They have used observed high resolution spectra to investigate whether Wing continuum band passes are influenced with other molecular absorption then updated the photometric system. In the updated system the continuum band passes are shifted to shorter wavelengths where the correlation between absorption strength and effective temperature shows less scatter. Based on Wings system, Ramsey also calibrated $\mathrm{TiO} \lambda 886 \mathrm{~nm}$ absorption band as a function of effective temperature (Ramsev 1981).

During 90's on one hand, extended TiO line lists were released which overcome the computational limitations especially in the visual region, on the other hand, large spectroscopic surveys made a vast amount of observed spectra of stars accessible to astrophysicist. These improvements have made it possible to search for $\mathrm{TiO}$ absorption bands in the visual region. We were motivated by Mavor et al. 2011, where they have searched for star's activity identification based on Ca II absorption using HARPS spectrograph at ESO. We have selected a list of $\mathrm{K}$ to $\mathrm{M}$ stars observed with KPNO $2.1 \mathrm{~m}$ telescope to investigate detectable $\mathrm{TiO}$ absorption in visual region. We used these spectra to make a calibration between $\mathrm{TiO}$ absorption and effective temperature. In section 2 we describe basic properties of $\mathrm{TiO}$ lines in 

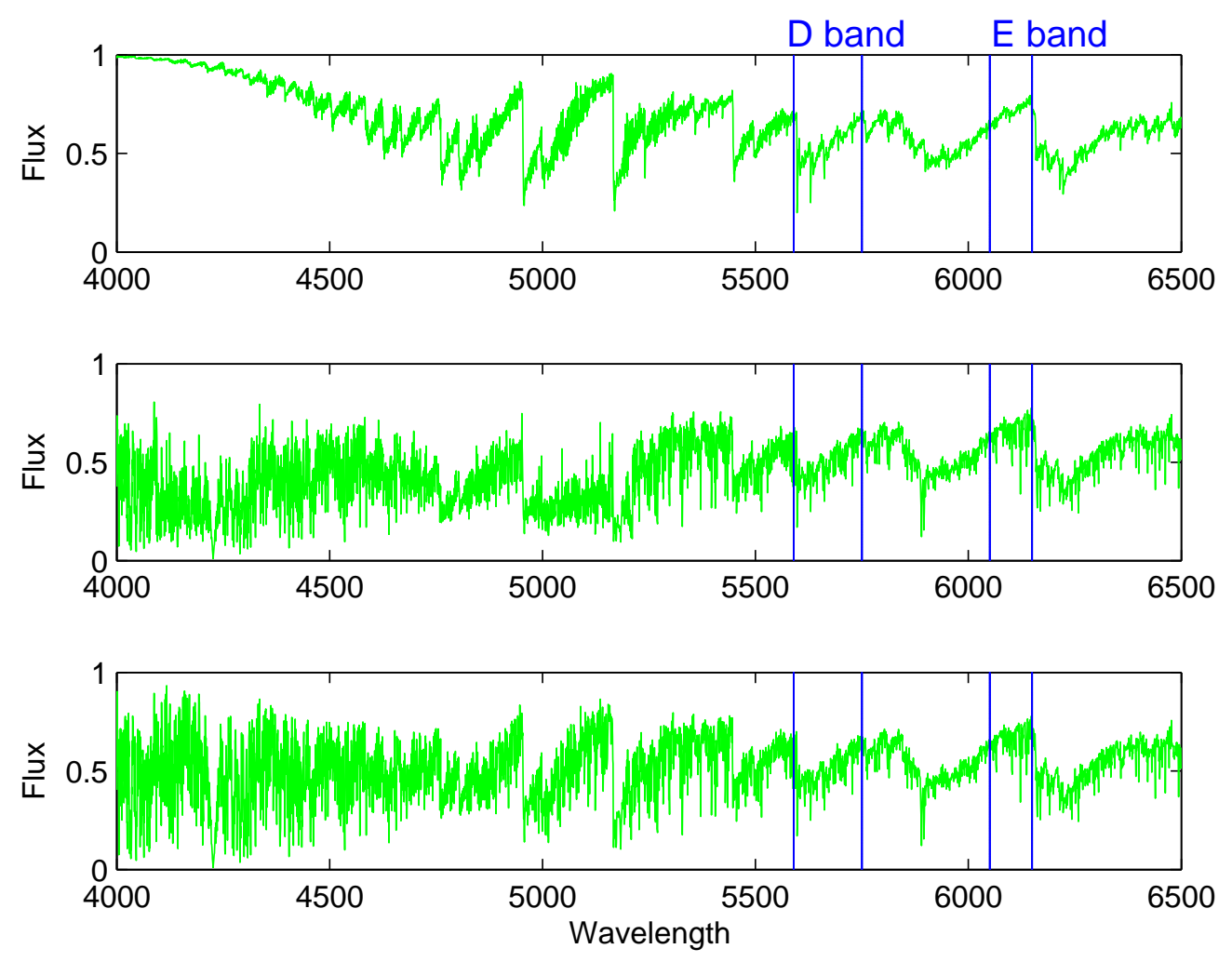

Figure 1. Synthetic Spectra of a star with $T_{\text {eff }}=3750 \mathrm{~K}$ and $\log g=3.0$ normalized to continume. The top panel shows absorption features belongs to $\mathrm{TiO}$ molecules with all other molecules and atoms omitted. In the middle panel contaminations produced by other molecules to $\mathrm{TiO}$ absorption bands are added. In the bottom panel all the molecules and atoms absorption lines are included. The selected band passes D and E are also shown on the panels.

visual region. In section 3 we introduce our index and later on section 4 we calibrated this index with effective temperature of K-M stars using their observed spectra.

\section{TIO ABSORPTION IN VISUAL WAVELENGTHS}

Although as a heavy metal, Titanium has tiny abundance in atmosphere of stars but Titanium Oxide is the most pervasive spectroscopic feature of cool stellar atmospheres in near infra-red region. Titanium Oxide molecule form in temperatures lower than $4000 \mathrm{~K}$. TiO opacity increases by decreasing temperature down to coolest atmosphere ever known then it can be used as a surface temperature indicator for spectral classes later than K (Wing 1992). TiO transitions between its ground and upper excited states in optical and near infrared wavelengths occurs mainly between electronic systems and can be divided into two groups: the first group are called allowed transitions and the strongest ones are $\gamma\left(A^{3} \Delta-X^{3} \Delta\right), \gamma^{\prime}\left(B^{3} \Pi-X^{3} \Delta\right)$ and $\alpha\left(C^{3} \Delta-X^{3} \Delta\right)$, in which all the A,B and $\mathrm{C}$ corresponds to excited levels with the same multiplicity as its ground state. The second group which can be referred as forbidden transitions are those occur in transitions between ground state and the non-equal multiplicity excited state. The strongest transitions of this group are: $\beta\left(c^{1} \Phi-a^{1} \Delta\right), \delta\left(b^{1} \Pi-a^{1} \Delta\right)$ and $\phi\left(b^{1} \Pi-d^{1} \Sigma^{+}\right)$
(Dobrodey 2001). The first group transitions are normally stronger than the second. Other transitions such as vibrational, rotational and transitions due to non-zero spin may influence TiO's spectrum marginally.

line lists calculated for $\mathrm{TiO}$ we found Schwenke's the most precise and suitable one for atmospheric modeling. He has computed a combined list completed to high energies (Allard. Hauschildt \& Schwenke 2000). This line lists includes 172 million lines. About 32 millions of them corresponds to four isotopes of $\mathrm{TiO}\left({ }^{50,49,47,46} \mathrm{TiO}\right)$, and 48 millions belongs to the most abundant one $\left({ }^{48} \mathrm{TiO}\right)$ (Allard. Hauschildt \& Schwenke 2000). Figure1 shows three synthetic spectra of a star with $T_{\text {eff }}=3750 \mathrm{~K}$ and $\log g=3.0$ calculated using ATLAS9 to show the effect of molecular and atomic absorption on the spectrum in visual wavelengths. We assumed local thermodynamic equilibrium (LTE) for the whole line forming region. Valenti, Piskunov \& Johns-Krull (1988) has checked that this is a good assumption for the great portion of line formation region although the absorption band core form in the high atmosphere where LTE is weak. To find a wide absorption feature capable of reproducing observable spectral index, we calculated a spectrum with all atomic and molecular absorption excluded except TiO.The top panel of figure 1 shows absorption features of the sole $\mathrm{TiO}$ molecules in visual region. In the middle panel we add other molecular 

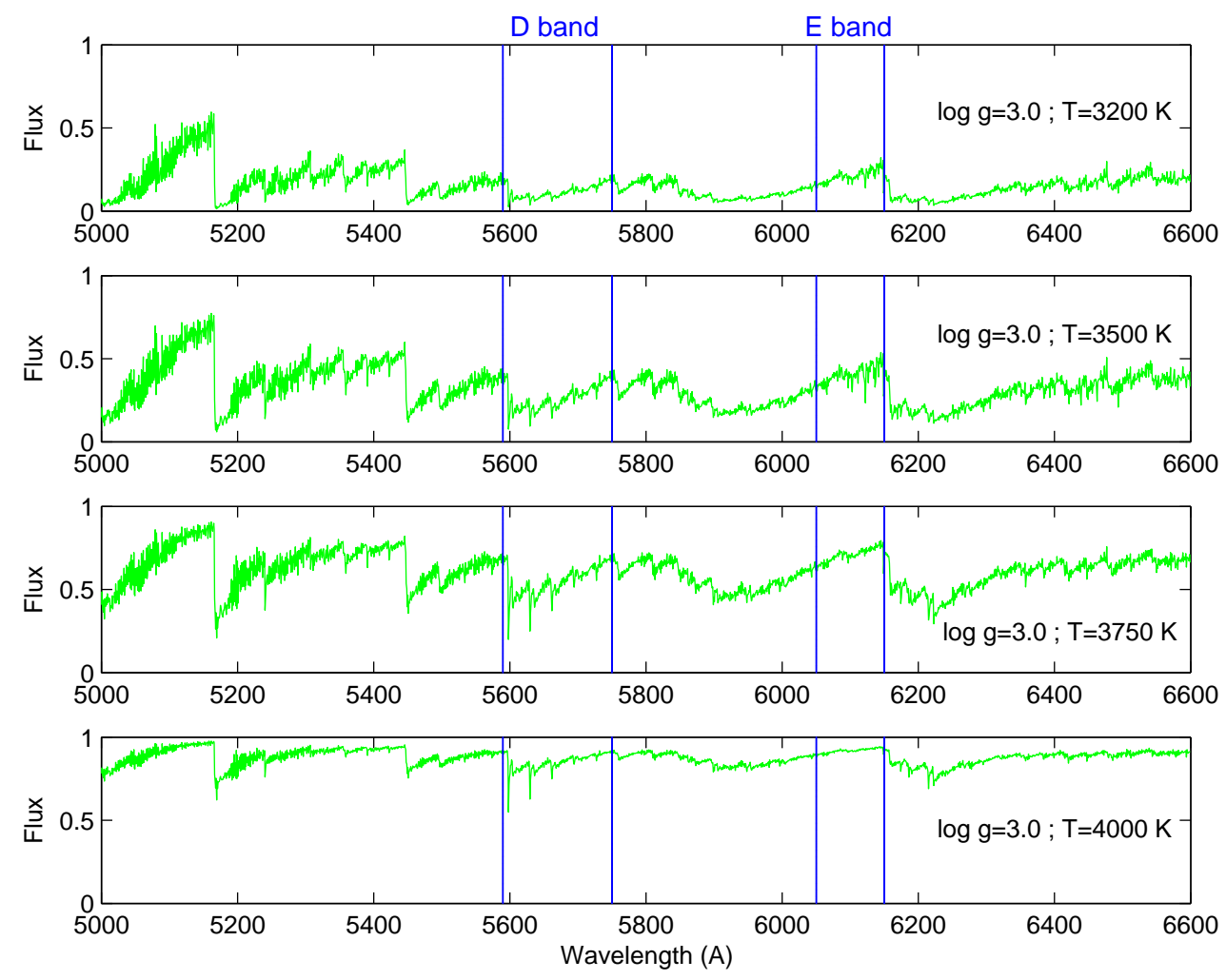

Figure 2. TiO absorption bands in four different temperatures with the same gravity. All the data has been produced using ATLAS 9 software. As it is obvious from the figure, TiO absorption bands deepens as temperature decreases declares that TiO's population increases as the star becomes cooler. The selected band passes D and E are also shown in the panels. In all four panels flux is normalized to continume. Filter D measures 559-575(nm).filter E measures 605-615(nm).

absorptions to the spectrum to explore possible contamination of $\mathrm{TiO}$ absorption systems. It is clear that $\mathrm{TiO}$ absorption bands in wavelengths shorter $5500 \mathrm{~nm}$ are strongly affected by presence of other molecules. As the middle panel shows the absorption system centered on $567 \mathrm{~nm}$ is less influenced and it can represent $\mathrm{TiO}$ absorption even in presence of other molecules. To be sure that atomic absorption will not destroy this system either, we finally turn on atomic absorption lines which is shown in the bottom panel. Figure 1 reveals that except the one at $567 \mathrm{~nm}$, other shorter absorption features of $\mathrm{TiO}$ are completely washed out by molecules like $\mathrm{H}-\mathrm{O}, \mathrm{CO}$ and $\mathrm{CN}$.

The effect of vanadium oxide molecules in visual part of spectrum is negligible. Kirkpatrick. Henry \& Mccarthy (1991) has listed absorption bands of this molecules with the shortest system at $733.4 \mathrm{~nm}$, which is far from our selected system. Finally we can conclude that the TiO absorption system at $567 \mathrm{~nm}$ is less contaminated by the molecular and atomic bands and could be used as a $\mathrm{TiO}$ absorption representative. This band is a $\beta(2-2)$ system of TiO 's 48 isotope consisting of 48 lines Valenti. Piskunov \& Johns-Krull 1988).

\section{VISUAL TIO INDEX}

A feasible spectral index which is going to represent abundance of a molecular junction or capable of calibrating a physical parameter like the effective temperature basically compares two region of the spectrum one deep inside the absorption region and the other far out on the continuum. The absorption feature might be wide enough to be observable with low resolution spectrographs or simple filter based photometers to admit long term dedicated observation with small telescopes. To explore the visual region for such $\mathrm{TiO}$ absorption we calculated atmospheric models for a grid of cool giant and dwarf stars using ATLAS 9 software package (Kurucz et al. 1991). The grid consists of atmospheric models ranging from $2900 \leqslant T_{\text {eff }} \leqslant$ $4200 K$ with steps $\Delta T=100 K$. The surface gravity was varies in the interval $3 \leqslant \log g \leqslant 5$ with steps $\Delta g=0.5$ (Castelli, Gratton \& Kurucz 1997). A number of 70 synthetic spectra were obtained. Figure 2 shows four spectra of this sample corresponds to $\log g=3.0$ with different effective temperatures. To search for a continuum band we can find at best only three regions to be labeled as continuum in visual wavelength in Figure 2 2 The first part (630$660) \mathrm{nm}$ is too contaminated to be labeled as continuum. The second part (520-540) nm seems to become noisy considering other molecules and atomic effect( Figure 1). As we need the continuum not to be crowded with other over- 


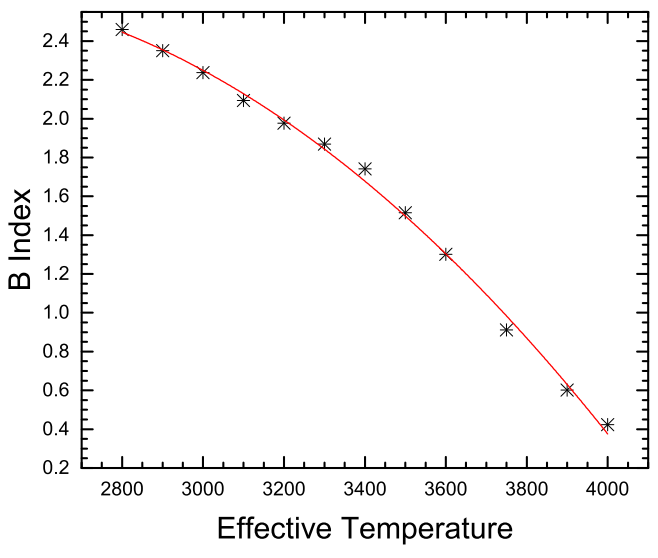

Figure 3. TiO B-index vs. effective temperature for synthetic spectra. all the data have been produced with ATLAS9.

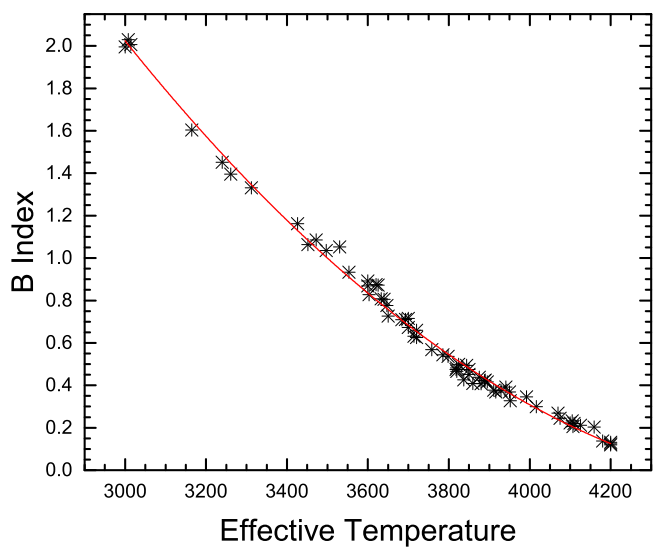

Figure 4. TiO B-index vs. effective temperature for observed spectra. All the information obtained from KPNO.It is clear that for real stars we observe a similar trend for the B-index as we have for synthesized spectra.

lapping $\mathrm{TiO}$ absorption bands, we cannot use this region due to some $\beta$ systems of $\mathrm{TiO}$ covers this part of spectrum (Valenti, Piskunov \& Johns-Krull 1988). It is also important to have a well-defined continuum near our absorption system to make the measurement of band head more precise.The third availabe continuum which is in(605-615) $\mathrm{nm}$ seems to be the most relevant one close to our porposed $\mathrm{TiO}$ band and free of other molecular absorption.

Following Wing's system, we introduced two new filters $\mathrm{D}$ and E. The central wavelengths and full width at halfmaximum band pass at each filters are listed in Table 1 Filter D measure the TiO absorption band strength centered at $567 \mathrm{~nm}$. The width of filter $\mathrm{D}$ is set to $16 \mathrm{~nm}$ which is widest possible to make use of whole absorption band. Filter $E$ is centered at $610 \mathrm{~nm}$ with a width of $10 \mathrm{~nm}$. A new visual Titanuim index which we call it "B-INDEX" can be formed by comparing integrated flux observed from these two band passes. By the way we define $B$ - index as

\begin{tabular}{lccc}
\hline Filter & Region Measured & $\begin{array}{c}\text { Central } \\
\text { wavelength } \\
(\mathrm{nm})\end{array}$ & $\begin{array}{c}\text { Bandpass } \\
\text { FWHM } \\
(\mathrm{nm})\end{array}$ \\
\hline $\mathrm{D}$ & TiO $\lambda 567 \mathrm{~nm}$ band & 567 & 16 \\
$\mathrm{E}$ & continum & 610 & 10 \\
\hline
\end{tabular}

Table 1. The B-index filter's system.

$B-$ index $=-2.5 \log \frac{\int F_{D}(\lambda) S_{D}(\lambda) d \lambda}{\int F_{E}(\lambda) S_{E}(\lambda) d \lambda}$

where $F_{D}(\lambda)$ and $F_{E}(\lambda)$ are integrated spectral flux in each band passes and $S_{D}(\lambda)$ and $S_{E}(\lambda)$ are appropriate filter response functions in each filter.

To demonstrate temperature sensitivity of the B-index, we assume a flat response function for both filters and calculate integrated flux in each filter for all synthesized spectra. Although the absorption band in $567 \mathrm{~nm}$ has a small signature of atomic absorption or possible molecular contamination, Figure 3 shows that the trend of variation of the index with effective temperature is smooth and monotonic.

To evaluate this index with observed spectra we selected a list of 78 stars with spectral index spanning from K2 to M8. These stars were observed with the Kitt Peak National Observatory (KPNO) $2.1 \mathrm{~m}$ telescope. The telescope was occupied with coudé Feed spectrograph with a F3KB CCD with pixel size of 15 micron which span visual wavelengths range from 346.5 to $946.9 \mathrm{~nm}$. They are listed in the first and fifth column of Table 2 Among these stars 12 was discarded due high level of noise or incomplete data. Effective temprature for these stars were found by searching on available observation or related literatures which is mentioned in fifth and tenth coloumn of Table 2 By the way the new defined B - index was calculated for all of these spectra to form a B-index-temperature calibration. Figure 4 shows that similar to synthesized spectra a smooth and monotonic decrease is seen which clearly shows that the new defined index has capability to recognize the effective temprature of cool atmospheres. To illustrate the calibration relation we fit a second order polynomial to the plot and found a simple calibration relation

$B-$ index $=6.667 \times 10^{-7} T_{\text {eff }}^{2}-0.0064 \times T_{\text {eff }}+15.17$

The standard deviation of the residuals around the fit is 0.0719. As it can be seen from the curve, the value of B-index is declining with increasing temperature. By decreasing temperature the $\mathrm{TiO}$ index increases up to a value about 2.00 which is corresponded to spectral type M7.5.

\section{DISCUSSION}

Figure 4 shows that the new B-index mimics the original TiO-index invented by Wing. The reader may ask why we need a visual representative for cool atmosphere of K-M stars where they are intrinsically faint in their visual spectrum. The original $\mathrm{TiO}$-index was adopted to the one of the strongest $\mathrm{TiO}$ absorption bands in the near infra-red where the spectrum is bright in $2900 \leqslant T_{\text {eff }} \leqslant$ 


\begin{tabular}{|c|c|c|c|c|c|c|c|c|c|}
\hline HD & TiO-Index (mag) & $T_{e f f}(\mathrm{~K})$ & Sp. Type & Ref. & HD & TiO-Index (mag) & $T_{e f f}(\mathrm{~K})$ & Sp. Type & Ref. \\
\hline 147379 & 0.3763 & 3929 & M1 & {$[3]$} & 20797 & 0.5686 & 3758 & M0 & {$[2]$} \\
\hline 29139 & 0.4520 & 3850 & K5 & [2] & 34255 & 0.3776 & 3911 & K4 & {$[5]$} \\
\hline 34334 & 0.1370 & 4180 & $\mathrm{~K} 2$ & {$[6]$} & 35620 & 0.1309 & 4200 & K3 & [10] \\
\hline 39225 & 0.6257 & 3720 & M2 & {$[7]$} & 39801 & 0.8077 & 3633 & M1-M2 & [11] \\
\hline 39853 & 0.4253 & 3837 & K5 & [10] & 40239 & 1.0526 & 3530 & M3 & {$[7]$} \\
\hline 42543 & 0.7095 & 3684 & M0 & {$[2]$} & 44478 & 0.8693 & 3600 & M3 & {$[9]$} \\
\hline 44537 & 0.5364 & 3798 & K5 & {$[1],[7]$} & 57651 & 0.6321 & 3714 & K5 & [10] \\
\hline 78712 & 1.6038 & 3165 & M6 & {$[10],[11]$} & 80493 & 0.4082 & 3880 & K7 & {$[10]$} \\
\hline 83787 & 0.4743 & 3818 & K6 & {$[2]$} & 97907 & 0.1237 & 4200 & K3 & [12] \\
\hline 99167 & 0.4813 & 3819 & K5 & {$[2]$} & 99998 & 0.2121 & 4125 & K3.5 & {$[1]$} \\
\hline 102212 & 0.4946 & 3844 & M1 & {$[16]$} & 108985 & 0.3691 & 3950 & K5 & {$[7]$} \\
\hline 111355 & 0.438 & 3875 & K5 & {$[2]$} & 112142 & 0.7764 & 3647 & M3 & {$[9]$} \\
\hline 112300 & 0.8754 & 3620 & M3 & [13] & 114961 & 2.0070 & 3014 & M7 & [10] \\
\hline 114287 & 0.3459 & 3992 & K5 & {$[2]$} & 118100 & 0.2230 & 4100 & K5 & {$[14]$} \\
\hline 120933 & 0.8721 & 3625 & K5 & {$[1]$} & 123657 & 1.0631 & 3452 & M4.5 & {$[15]$} \\
\hline 126327 & 1.9960 & 3000 & M7.5 & [15] & 123934 & 0.544 & 3785 & M1 & {$[1],[2]$} \\
\hline 128000 & 0.3918 & 3941 & K5 & {$[17]$} & 131918 & 0.2056 & 4107 & K4 & {$[5],[2],[16],[17],[18],[4]$} \\
\hline 136726 & 0.2034 & 4159 & K4 & {$[20]$} & 138481 & 0.4247 & 3890 & K5 & {$[19]$} \\
\hline 139669 & 0.4161 & 3895 & K5 & {$[20]$} & 175588 & 1.0856 & 3472 & M4 & {$[1]$} \\
\hline 148513 & 0.2447 & 4075 & K4 & {$[10]$} & 148783 & 1.3954 & 3261 & M6 & {$[1]$} \\
\hline 149161 & 0.3277 & 3952 & K4 & {$[16]$} & 158899 & 0.22 & 4109 & K4 & {$[22]$} \\
\hline 167006 & 0.8054 & 3640 & M3 & [4] & 168720 & 0.4663 & 3820 & M1 & {$[1]$} \\
\hline 180928 & 0.2322 & 4105 & $\mathrm{k} 4$ & {$[1],[2],[5]$} & 169305 & 0.7149 & 3700 & M2 & {$[1],[7]$} \\
\hline 177940 & 2.0292 & 3008 & M7 & [1] & 186619 & 0.4220 & 3888 & M0 & {$[1],[2]$} \\
\hline 189319 & 0.3683 & 3916 & M0 & [10] & 191372 & 0.7264 & 3650 & M3 & {$[7]$} \\
\hline 196610 & 1.4513 & 3240 & M6 & {$[7]$} & 197812 & 1.3308 & 3312 & M5 & [1] \\
\hline 196777 & 0.6598 & 3720 & M1 & {$[7]$} & 197939 & 0.8274 & 3603 & M3 & {$[5]$} \\
\hline 200527 & 0.9327 & 3553 & M4 & [1] & 202259 & 0.4990 & 3824 & M1 & {$[1],[5]$} \\
\hline 203535 & 0.4093 & 3859 & M0 & {$[1]$} & 204445 & 0.7110 & 3693 & M1 & {$[1],[7]$} \\
\hline 206936 & 0.6733 & 3700 & M2 & [19] & 207134 & 0.1195 & 4200 & K3 & {$[12]$} \\
\hline 217906 & 0.8917 & 3600 & M2 & [10] & 221615 & 1.1615 & 3426 & M5 & {$[1]$} \\
\hline 225212 & 0.2988 & 4016 & K3 & {$[22],[28]$} & 237903 & 0.2694 & 4070 & $\mathrm{~K} 7$ & [17] \\
\hline 146051 & 0.4751 & 3850 & M0.5 & [21] & 172816 & 1.0358 & 3497 & M4 & {$[10]$} \\
\hline
\end{tabular}

Table 2. List of stars. References: [1] Rimoldini et al. (2012); [2] Gontcharov (2006); [3] Shava \& Olling $\quad(2011) ; \quad[4]$ McDonald, Ziilstra \& Bover (2012); [5] Bobvlev, Goncharov \& Baikova (2006); [6] Ofek (2008); [7] Tevssier et al. (2012); [8] Ramirez \& Melendez (2005); [9] Smith, Price \& Baker (2004); [10] Blum, Ramirez \& Sellgren (2003); [11] Glebocki \& Gnacinsk (2005); [12]Le Borgne et al. (2003); [13] Salim \& Gould (2003); [14] Cenarro et al. (2007) ; [15] Price et al. (2010) ; [16] Koleva \& Vazdekis (2012) ; [17] Anderson \& Francis (2012) ; [18] Sanchez-Blazquez et al. (2006) ;[19] Eggleton \& Tokovinin (2008) ; [20] Massarotti et al. (2008) ; [21] Tabur, Kiss \& Bedding (2009) ; [22] Wallace et al. (2000).

$4200 K$. Mirtorabi, Wasatonic \& Guinan (2003) have shown that TiO-index can reveal stellar activity of F-G type stars. They observed $\lambda$ And in near infra-red and detected $\mathrm{TiO}$ absorption through nonzero and variable Wing TiO-index. The quiet photosphere of $\lambda$ And is too hot to have TiO molecule. It is believed that part of the variability of this star comes from large coverage of unevenly distributed spots. The absorption might come from this cool active regions. They also found that $\mathrm{TiO}$ molecules can survive even when the star is brightest which means part of the active regions might distributed evenly on the surface of the star with no observable effects on visual light curve. A visual $\mathrm{TiO}$ index can represent this activity more precisely. In future work we are going to use the B-index to search for stellar activity in visual spectrum of $\mathrm{F}$ and $\mathrm{G}$ stars where they are more brighter in visual than infra-red.

\section{REFERENCES}

Anderson E., Francis C., 2012, Astronomy Letters, 38, 331
Allard F., Hauschildt P.H., Schwenke D., 2000, AJ, 540, 1005

Azizi F., Mirtorabi M.T., 2015, Ap\&SS, 357, 96A

Blum R.D., Ramirez S.V., Sellgren K., 2003, ApJ, 597, 323

Bobylev V.V., Goncharov G.A., Bajkova A.T., 2006, Astronomy Reports, 50, 733

Castelli F., Gratton R.G., Kurucz R.L., 1997, A\& A, 318, 841

Cenarro A.J., Peletier R.F., Sanchez-Blazquez P., Selam S.O., Toloba E., Cardiel N., Falcon-Barroso J., Gorgas J., Jimenez-Vicente J., Vazdekis A., 2007,MNRAS, 374, 664 Dobrodey N. V., 2001, A\&A, 365, 642

Eggleton P.P., Tokovinin A.A., 2008, MNRAS, 389, 869

Glebocki R., Gnacinski P., 2005, VizieR Online Data Catalog, 3244, 0

Gontcharov G. A., 2006, Astronom Letters, 32, 759

Kirkpatrick J.D., Henry T.J., Mccarthy D.W., 1991, ApJ, 77,417

Koleva M., Vazdekis A., 2012, A\&A, 538

Kurucz, R.L., Davis, P., Upgren, A., Janes, K., (1991) Precision photometry: Astrophysics of galaxy workshop, New 
York, p.27,New lines, new models, new colors

Kurucz R.L., Davis P., Upgren A., Janes K., 1991,ApJ, 245, p. 27

Le Borgne J.F., Bruzual G., Pello R., Lancon A., RoccaVolmerane B., Sanahuja B., Schaerer D., Soubrian C., Vilchez-Gomez R., 2003, A\& A, 402, 433

Massarotti A., Latham D.W., Stefanik R.P., Fogel J., 2008, AJ, 135, 209

Mayor M., Lovis C., Dumusque X., Santos N.C., Bouchy F., Pepe M., Queloz D., Segransan D., Udry S., Marmier M., Benz W., Bertaux J.L., 2011, A\& A, 498, 851

McDonald L., Zijlstra A.A., Boyer M.L., 2012, MNRAS, 427, 343

Mirtorabi M.T., Wasatonic R., Guinan E. F., 2003, AJ, 125,3265

Ofek E. O., 2008, MNRAS, 1128

Price S.D., Smithl B.J., Kuchar T.A., Mizuno D.R., Kramer K., 2010, ApJ, 190, 203

Ramirez I., Melendez J., 2005, ApJ, 626, 446

Ramsey L. W., 1981, AJ, 86, 557

Rimoldini L., Dubath P., Suveges M., Lopez M., Sarro L.M., Blomme J., DeRidder J., Cuypers J., Guy L., Mowlavi N., Lecoueur-Taibi l., Beck M., Jan A., Nienartowicz K., Ordonez-Blanco D., Lebzelter T., Eyer L., 2012, MNRAS, 427, 2917

Robinson K., 1981, Spectroscopy: The key to the stars, Springer-Verlag,London

Salim S., Gould A., 2003, ApJ, 582, 1011

Sanchez-Blazquez P., Peletier R.F., Selam S.O., Cardiel N., Falcon-Barroso J., Gorgas J., Jimenez-Vicente J., Vazdekis A, 2006, MNRAS, 371, 703

Shaya E.J., Olling R.P., 2011, ApJ, 192

Smith B.J., Price S.D., Baker R.I., 2004, ApJ, 154, 673

Tabur V., Kiss L.L., Bedding T.R., 2009, ApJ, 703, 72

Teyssier D., Quintana-Lacaci G., Marston A.P., Bujarrabal V., Alcolea J., Cernicharo J., Decin L., Dominik C., Justtanont K., de Koter A., Melnick G., Menten K.M., Neufeld D.A., Olofsson H., Planesas P., Schmidt M., Soria-Ruiz R., Schoier F.L., Szczerba R., Waters L.B.F.M,, 2012, A\&A, 545

Valenti J. A., Piskunov N., Johns-Krull C. M., 1998, ApJ, 498, 851

Wallace L., Meyer M.R., Hinkle K., Edwards S., 2000, ApJ, 535,325

Wing R.F., 1992, JAVSO, 21, 42 